\title{
Baixo letramento em saúde: uma realidade brasileira
}

\section{Hevist国}

\author{
CARTA AO EDITOR
}

Low health literacy: a Brazilian reality

Josué da Silva Brito ${ }^{1}$ Christiano Tadeu Sanches Mattos Kilesse ${ }^{1,2}$ Priscilla Itatianny de Oliveira Silva ${ }^{1}$
Lemos com muita atenção o estudo realizado por Pinhati et $\mathrm{al}^{1}$, no qual não se encontrou associação entre o baixo letramento em saúde (BLS) e a pressão arterial não controlada no nível secundário de atenção à saúde. Primeiramente, congratulamos os autores por inovarem investigando o fator no nível secundário de atenção à saúde.

A adesão ao tratamento depende de um conjunto de fatores, incluindo os aspectos socioeconômicos e comportamentais do indivíduo, particularidades da doença e do tratamento, também depende das posturas adotadas pelo profissional de saúde e de aspectos do sistema sanitário. Fatores como emprego, estabilidade financeira, casamento, crença de que o tratamento é o correto e eficaz para condição e apoio social apresentam associação positiva com o uso regular de medicamento e controle de doenças crônicas. Já os extremos de idade, isto é, pacientes muito jovens ou muito idosos, transtornos do humor, altos custos, efeitos colaterais, polifarmácias e distúrbios da memória apresentam associação negativa. ${ }^{2-4}$

Miller ${ }^{5}$, em metanálise, demonstrou que a alfabetização em saúde se associa positivamente a adesão terapêutica, principalmente quando relacionada a doenças cardiovasculares. O risco de não adesão em pacientes com baixo letramento em saúde, fator apontado pela Organização Mundial da Saúde como um dos determinantes na adesão ao tratamento, ${ }^{4}$ foi $16 \%$ maior. A educação em saúde se dá de forma mais eficaz, segundo o mesmo autor, quando o próprio paciente classifica seu nível educacional em saúde.

Choi et al6, em estudo realizado com 1523 pacientes, encontraram relação entre boa adesão a medicação anti-hipertensiva e idade $\geq 65$ anos, exercício físico regular ao menos 1 vezes por semana, hospital localizado em região metropolitana, histórico familiar de doenças cardiovasculares ou hipertensão, uso de duas ou mais classes de anti-hipertensivos e uso concomitante de medicamentos para diabetes mellitus.

Em nossa experiência, obtida na atuação na Estratégia de Saúde da Família, em comunidades carentes através de projetos sociais e de extensão e também na atuação nos níveis secundários e terciários, observamos, no geral, baixo nível de conhecimentos em saúde, algo intimamente ligado a menor renda e menor escolaridade. Muitos pacientes carregam consigo crenças devastadoras para a saúde, mas muito comuns em nossa região, como "o uso de medicamento diário causa vício", "só é preciso utilizar o anti-hipertensivo quando sinto dor de cabeça", "usar o medicamento uma vez a cada dois dias tem o mesmo efeito".

Esclarecer os pacientes, principalmente os com idade avançada, é um desafio para todos os níveis de saúde. Apesar das repetitivas tentativas de auxiliar o paciente em compreender seu estado de saúde, a hipertensão, muita das vezes, não é vista como uma doença grave. O acidente vascular cerebral é grave, o infarto agudo do miocárdio também é grave, mas a hipertensão arterial, na visão popular, não. A ligação entre a hipertensão e suas complicações é ignorada ou desconhecida.

O estudo de Pinhati et al $^{1}$ não nega a associação do baixo conhecimento em saúde, que surpreende por apresentar prevalência superior a $70 \%$, com a hipertensão não controlada, mesmo que não a tenha encontrado, resultado que os autores justificam como possivelmente oriundo das características demográficas e sociais da amostra. Indiretamente, a alta prevalência permite aferir que o BLS seja, talvez, um problema subdimensionado. Portanto, reforça a necessidade de se educar em saúde.

Planos de tratamento elaborados, medidas multiprofissionais, tudo se perde se houver incompreensão por parte do paciente. Atender a integralidade e promover a equidade são princípios fundamentais da saúde brasileira assentados na Constituição Federal de $1988 .^{7}$ Para tanto, é preciso que a educação em saúde seja inclusiva e ultrapasse a academia, atingindo a todos que carecem de cuidados.

\section{REFERÊNCIAS}

1. Pinhati RR, Tavares PL, Marsicano EO, Fernandes NS, Colugnati $F A$, et al. Baixo letramento em saúde em pacientes idosos com pressão arterial não controlada em nível secundário de atenção à saúde. HU Rev. 2019; 45(1):13-21. DOI: 10.34019/1982-8047.2019.v45.16970

2. Gast A, Mathes T. Medication adherence influencing factors-an (updated) overview of systematic reviews. Syst Rev. 2019; 8(1):112. Published 2019 May 10. doi:10.1186/s13643-0191014-8

3. Jaam M, Ibrahim MIM, Kheir N, Awaisu A. Factors associated with medication adherence among patients with diabetes 
in the Middle East and North Africa region: a systematic mixed studies review. Diabetes Res Clin Pract. 2017; 129:1-15. doi: 10.1016/j.diabres.2017.04.015.

4. Sabaté E (ed.) Adherence to Long-term therapies: evidence to action. Geneva: World Health Organization; 2003.

5. Miller TA. Health literacy and adherence to medical treatment in chronic and acute illness: a meta-analysis. Patient Educ Couns. $2016 ; 99(7): 1079-86$. doi:10.1016/j.pec.2016.01.020

6. Choi HY, Oh IJ, Lee JA, Lim J, Kim YS et al. Factors Affecting Adherence to Antihypertensive Medication. Korean J Fam Med. 2018; 39(6):325-32. doi:10.4082/kjfm.17.0041

7. Brasil. Constituição da República Federativa do Brasil. Brasília: Senado; 1988.

${ }^{1}$ Faculdade de Medicina, Centro Universitário Atenas, Brasil.

${ }^{2}$ Faculdade de Odontologia, Pontifícia Universidade Católica de Minas Gerais, Brasil.

\footnotetext{
$\square$ Josué da Silva Brito

Rua das Mansões, 2010, Esplanada, Paracatu, Minas Gerais

CEP: $38608-230$

円 josuedasilvabrito@gmail.com
} 